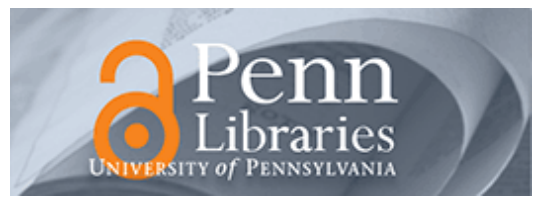

University of Pennsylvania ScholarlyCommons

8-18-2008

\title{
Can the Imaginary Part of Permeability be Negative?
}

Vadim A. Markel

University of Pennsylvania, vmarkel@mail.med.upenn.edu

Follow this and additional works at: https://repository.upenn.edu/be_papers

Part of the Biomedical Engineering and Bioengineering Commons

\section{Recommended Citation}

Markel, V. A. (2008). Can the Imaginary Part of Permeability be Negative?. Retrieved from https://repository.upenn.edu/be_papers/174

Suggested Citation:

Markel, V.A. (2008). "Can the imaginary part of permeability be negative?" Physical Review E. 78, 026608.

(c) 2008 The American Physical Society

http://dx.doi.org/10.1103/PhysRevE.78.026608

This paper is posted at ScholarlyCommons. https://repository.upenn.edu/be_papers/174

For more information, please contact repository@pobox.upenn.edu. 


\title{
Can the Imaginary Part of Permeability be Negative?
}

\author{
Abstract \\ When new composite optical materials are developed experimentally or studied in numerical simulations, \\ it is essential to have a set of fundamental constraints that the optical constants of such materials must \\ satisfy. In this paper I argue that positivity of the imaginary part of the magnetic permeability may not be \\ one of such constraints, particularly in naturally occurring diamagnetics and in artificial materials that \\ exhibit diamagnetic response to low-frequency or static magnetic fields.

\section{Disciplines} \\ Biomedical Engineering and Bioengineering | Engineering

\section{Comments} \\ Suggested Citation: \\ Markel, V.A. (2008). "Can the imaginary part of permeability be negative?" Physical Review E. 78, 026608. \\ (C) 2008 The American Physical Society \\ http://dx.doi.org/10.1103/PhysRevE.78.026608
}




\title{
Can the imaginary part of permeability be negative?
}

\author{
Vadim A. Markel* \\ Departments of Radiology and Bioengineering, University of Pennsylvania, Philadelphia, Pennsylvania 19104, USA
}

(Received 25 February 2008; published 18 August 2008)

\begin{abstract}
When new composite optical materials are developed experimentally or studied in numerical simulations, it is essential to have a set of fundamental constraints that the optical constants of such materials must satisfy. In this paper I argue that positivity of the imaginary part of the magnetic permeability may not be one of such constraints, particularly in naturally occurring diamagnetics and in artificial materials that exhibit diamagnetic response to low-frequency or static magnetic fields.
\end{abstract}

DOI: 10.1103/PhysRevE.78.026608

PACS number(s): 41.20.Jb, 75.20.-g, 78.20.-e

\section{INTRODUCTION}

In this paper, I argue that there is no solid theoretical basis to believe that the imaginary part of the magnetic permeability is always positive for any frequency and any material. I will further argue that, in the case of diamagnetics [1], there is a strong indication to the contrary.

The paper is motivated by a recent controversy [2,3] which touched on the subject of whether the imaginary parts of the permittivity $\epsilon(\omega)=\epsilon^{\prime}(\omega)+i \epsilon^{\prime \prime}(\omega)$ and permeability $\mu(\omega)=\mu^{\prime}(\omega)+i \mu^{\prime \prime}(\omega)$ of a material can ever be negative. The controversy was, in turn, sparked by the publication of Ref. [4] by Koschny et al. in which numerical simulations of the effective parameters of an artificial composite optical medium have been reported. In particular, it was claimed that both $\epsilon^{\prime \prime}$ and $\mu^{\prime \prime}$ can be negative in some frequency ranges. Koschny et al. and the authors of the subsequent comments $[2,3]$ have relied in their arguments on a standard expression for the rate at which the energy of a monochromatic electromagnetic field of frequency $\omega$ is dissipated into heat at a point $\mathbf{r}$ inside a spatially uniform medium. I will refer to this quantity simply as the heating rate and denote it by $q(\mathbf{r})$. The standard expression for the heating rate is [5]

$$
q(\mathbf{r})=\frac{\omega}{8 \pi}\left[\epsilon^{\prime \prime}(\omega)\left|\mathbf{E}_{\omega}(\mathbf{r})\right|^{2}+\mu^{\prime \prime}(\omega)\left|\mathbf{H}_{\omega}(\mathbf{r})\right|^{2}\right],
$$

where $\mathbf{E}_{\omega}$ and $\mathbf{H}_{\omega}$ are the complex amplitudes of the electric and magnetic fields while the real-valued fields are obtained from $\mathbf{E}(\mathbf{r}, t)=\operatorname{Re}\left[\mathbf{E}_{\omega}(\mathbf{r}) \exp (-i \omega t)\right]$ and analogously for the magnetic field. Note that formula (1) should not be confused with the expression for the energy density $W(\mathbf{r})$ stored in the medium which is discussed, for example, in Ref. [6] [see Eqs. (4) and (5) of this reference]. The latter quantity can be defined only approximately in the spectral region where the material is nearly transparent ([5], Sec. 80). In contrast, $q(\mathbf{r})$ is well defined for arbitrary dispersion; its integral over the body volume gives the absorption cross section which is a well-defined thermodynamic quantity.

Koschny et al. did not dispute that $q(\mathbf{r})$ must be positive but argued that there is no requirement that $\epsilon^{\prime \prime}(\omega)$ and $\mu^{\prime \prime}(\omega)$ be positive simultaneously; instead, they suggested that only the sum of the two terms in the right-hand side of Eq. (1) is

\footnotetext{
*vmarkel@mail.med.upenn.edu
}

required to be positive. The main point of the comments $[2,3]$ was that it is possible to envision a geometrical arrangement in which either one of these two terms is arbitrarily small while $\epsilon^{\prime \prime}(\omega)$ and $\mu^{\prime \prime}(\omega)$ are properties of the medium and, therefore, are independent of the geometry. Therefore, both $\epsilon^{\prime \prime}(\omega)$ and $\mu^{\prime \prime}(\omega)$ must be positive independently of each other to ensure the positivity of $q(\mathbf{r})$ for all possible medium geometries and illumination patterns. In their reply to the above two comments, Koschny et al. have argued that what they retrieve are some "effective" parameters of a periodic structure which are wave vector dependent and that such parameters are not subject to the same constraints as those of homogeneous materials. Koschny et al. did not indicate whether they think that the same reasoning applies to materials which exhibit spatial dispersion and did not reflect any further on the generality of Eq. (1). Simulations in which negative values of either $\epsilon^{\prime \prime}(\omega)$ or $\mu^{\prime \prime}(\omega)$ are reported continue to appear in the literature [7].

The purpose of this paper is to point out that one encounters a serious difficulty when the requirement $\mu^{\prime \prime}(\omega)>0$ is imposed. Specifically, it is difficult to reconcile the analytical properties of $\mu(\omega)$, the above inequality and the existence of diamagnetics. In fact, this difficulty is known. In particular, it is discussed, although somewhat indirectly, in Electrodynamics of Continuous Media by Landau and Lifshitz [5]. It is suggested in this text that the Kramers-Kronig relations for $\mu(\omega)$ must be modified [as compared to those for $\epsilon(\omega)$ ] to allow for the existence of diamagnetics. This argument is reproduced below. However, the justification for this procedure is hardly satisfying; in particular, it results in an expression for $\mu^{\prime}(\omega)$ which has an incorrect high-frequency asymptote. In what follows, I will argue that the proofs of positivity given in the comments $[2,3]$ fully apply to $\epsilon^{\prime \prime}$, but not to $\mu^{\prime \prime}$. In other words, I think that the possibility is open for $\mu^{\prime \prime}$ to be negative in diamagnetics without violating any of the fundamental laws of nature. If this is indeed so, the need for the modification of the Kramers-Kronig relations for $\mu$ disappears and a more logical and self-consistent theory results.

\section{SIGN OF $\mu^{\prime \prime}(\omega)$ AND CAUSALITY}

The nature of the difficulty noted above is quite elementary. Consider the standard Kramers-Kronig relation written 
without modification (that is, without the modification of Landau and Lifshitz) as

$$
\mu^{\prime}(\omega)=1+\frac{2}{\pi} \int_{0}^{\infty} \frac{x \mu^{\prime \prime}(x)}{x^{2}-\omega^{2}} d x .
$$

Set $\omega=0$ in the above equation to obtain

$$
\mu^{\prime}(0)=1+\frac{2}{\pi} \int_{0}^{\infty} \frac{\mu^{\prime \prime}(\omega)}{\omega} d \omega .
$$

It follows from Eq. (3) that the condition $\mu^{\prime}(0)<1$ which is commonly encountered in diamagnetics is incompatible with $\mu^{\prime \prime}(\omega)>0 \forall \omega>0$.

I will now translate directly from the 1982 Russian edition of Electrodynamics of Continuous Media, Sec. 82 (comments in the square brackets are mine): "All the results obtained above [for the dielectric permittivity $\epsilon(\omega)$ ] are also applicable (with a minor modification) to the magnetic permeability $\mu(\omega)$. The difference is related, in the first place, to the fact that, when the frequency is increased, the function $\mu(\omega)$ ceases to have physical meaning at relatively small values of $\omega$. Thus, for example, the Kramers-Kronig formulas should be applied to $\mu(\omega)$ as follows. Consider a finite, rather than infinite, interval or $\omega$ (from 0 to $\omega_{1}$ ) such that the function $\mu(\omega)$ still has physical meaning near the upper bound of this interval, yet it is constant [for $\omega \approx \omega_{1}$ ] and its imaginary part can be assumed to be zero; the corresponding real part of $\mu$ is denoted by $\mu_{1}$. Then the equation (82.8) [identical to Eq. (2) in this text but written for $\epsilon(\omega)$ ] should be written as

$$
\mu^{\prime}(\omega)=\mu_{1}+\frac{2}{\pi} \int_{0}^{\omega_{1}} \frac{x \mu^{\prime \prime}(x)}{x^{2}-\omega^{2}} d x .
$$

In contrast to $\epsilon_{0}, \mu_{0}=\mu(0)$ can be either larger or smaller than unity."

As was mentioned above, the arguments in favor of this modification of the Kramers-Kronig formula are not satisfying. Several objections can be raised.

(i) First, the interpretation of the statement that "the function $\mu(\omega)$ ceases to have physical meaning at relatively small values of $\omega$ " is not clear. Perhaps a more accurate statement would be the following: there is no physical meaning in introducing a function $\mu(\omega)$ that is different from unity at frequencies which are larger than a certain threshold. In other words, the function $\mu(\omega)$ must asymptotically approach unity at relatively small frequencies and any theory in which $\mu(\omega)$ is taken to be different from unity, e.g., at optical frequencies, is unphysical. This interpretation is consistent, with the statement contained in Sec. 79 of Electrodynamics of Continuous Media, namely, "Unlike $\epsilon(\omega), \mu(\omega)$ ceases to have physical meaning at relatively small frequencies; an account of the difference $\mu(\omega)-1$ at those frequencies would amount to an unlawful correction [that is, exceeding precision of the theory]." Of course, at sufficiently large frequencies, macroscopic Maxwell equations are inapplicable and in this limit both $\epsilon$ and $\mu$ have no physical meaning. However, it is logical to assume that, before this happens, both functions $\epsilon(\omega)$ and $\mu(\omega)$ must approach unity and integrals of the type (2) must converge. Thus, while the upper limit of the integration in (2) is infinity, it is not practical to integrate any experimentally measured functions up to the frequencies that correspond, for example, to $\gamma$ rays. The primary difference between $\epsilon$ and $\mu$ is that $\mu(\omega)$ approaches unity at much smaller frequencies than $\epsilon(\omega)$. But, if this is so, one can choose $\mu_{1}=1$ and set formally $\omega_{1}=\infty$ in (4) with the understanding that the integral converges at the upper limit and should not be extended in a numerical calculation beyond a certain frequency for which the macroscopic equations are still valid.

(ii) Assume that (4) with $\mu_{1} \neq 1$ is correct. That means that at frequencies $\omega>\omega_{1}$, the permeability is different from unity and does not change, as long as $\mu(\omega)$ has a physical meaning. However, it is known that at sufficiently high frequency, say $\omega_{2}>\omega_{1}$, the value of $\mu\left(\omega_{2}\right)$ must be unity. Consequently, something happens in the interval $\omega_{1}<\omega<\omega_{2}$ : either $\mu(\omega)$ is changed (contrary to the original assumption) or it ceases to have "physical meaning" abruptly at some intermediate frequency. In other words, (4) has an incorrect high-frequency asymptote.

I believe that the above two objections are sufficiently disturbing to reexamine the requirement $\mu^{\prime \prime}(\omega)>0$. To this end, consider in more detail the arguments in favor of the above inequality contained in the comments $[2,3]$.

\section{SIGN OF $\mu^{\prime \prime}(\omega)$ AND THE SECOND LAW OF THERMODYNAMICS}

In their comment, Depine and Lakhtakia [2] have applied the condition that the function $q(\mathbf{r})$ be positive at each point inside the material. Since there might be points $\mathbf{r}$ such that either $\mathbf{E}_{\omega}(\mathbf{r})=\mathbf{0}$ or $\mathbf{H}_{\omega}(\mathbf{r})=\mathbf{0}$ (rectangular waveguide modes are quoted as an example), one immediately arrives at the conclusion that $\epsilon^{\prime \prime}(\omega)$ and $\mu^{\prime \prime}(\omega)$ must be positive simultaneously and independently of each other. However, the pointwise positivity of $q(\mathbf{r})$ does not really follow from the second law of thermodynamics. The latter requires that the total entropy of a closed system be increased in any thermodynamically irreversible process. The second law does not say that the entropy cannot be decreased locally (otherwise, refrigeration would be impossible). One can also apply the alternative formulation of the second law, namely, that perpetual motion machine of the second kind is impossible. Assume that $q(\mathbf{r})<0$ in a very small vicinity of the point $\mathbf{r}_{0}$ inside the medium. As long as the total absorption (per unit time)

$$
Q=\int_{V} q(\mathbf{r}) d^{3} r
$$

is positive, there is no practical way to utilize the local negativity of $q(\mathbf{r})$ for constructing a perpetual motion machine. Therefore, the arguments of Depine and Lakhtakia appear to be incomplete and not immune to challenges.

In the second comment [3], Efros suggests scenarios in which one of the terms $\epsilon^{\prime \prime}(\omega)\left|\mathbf{E}_{\omega}(\mathbf{r})\right|^{2}, \mu^{\prime \prime}(\omega)\left|\mathbf{H}_{\omega}(\mathbf{r})\right|^{2}$ in Eq. (1) is much smaller than the other at every point $\mathbf{r}$ inside the material. This is significant because, if there are conditions 
under which either of these terms can be neglected at every point $\mathbf{r}$ (inside the material), then the positivity of the integral quantity $Q$ given by (5) explicitly requires that $\epsilon^{\prime \prime}$ and $\mu^{\prime \prime}$ be positive independently of each other. While I do not doubt that it is easy to achieve the condition $\epsilon^{\prime \prime}(\omega)\left|\mathbf{E}_{\omega}(\mathbf{r})\right|^{2}$ $\gg \mu^{\prime \prime}(\omega)\left|\mathbf{H}_{\omega}(\mathbf{r})\right|^{2}$ in the whole range of experimentally relevant frequencies (e.g., by placing a small object between the plates of a capacitor as was suggested by Efros), it is not as clear that $\epsilon^{\prime \prime}(\omega)\left|\mathbf{E}_{\omega}(\mathbf{r})\right|^{2}<\mu^{\prime \prime}(\omega)\left|\mathbf{H}_{\omega}(\mathbf{r})\right|^{2}$ can always be achieved, particularly in the case of diamagnetics. This is discussed in more detail below.

As suggested by Efros, let us place a small but macroscopic object (a sphere for simplicity) on the axis of a solenoid to which an alternating voltage of the frequency $\omega$ is applied. The solenoid will create a (nearly) spatially uniform magnetic field $\mathbf{H}_{\omega}$. The electric field inside the solenoid will also exist at any finite frequency due to the induction. As is well known, the magnitude of the electric field can be estimated in this case as $E_{\omega} \sim(1 / 2)(\omega R / c) H_{\omega}$, where $R$ is the characteristic size of the object, say, the sphere's radius. We thus have the following estimate:

$$
\frac{\epsilon^{\prime \prime}(\omega)\left|\mathbf{E}_{\omega}(\mathbf{r})\right|^{2}}{\mu^{\prime \prime}(\omega)\left|\mathbf{H}_{\omega}(\mathbf{r})\right|^{2}} \sim \frac{1}{4}\left(\frac{\omega R}{c}\right)^{2} \frac{\epsilon^{\prime \prime}(\omega)}{\mu^{\prime \prime}(\omega)} .
$$

While the factor $\omega R / c$ can be small at sufficiently small frequencies (practically, this factor can be less than unity for $R \sim 10 \mathrm{~nm}$ even in the optical range; it is, however, difficult to imagine a solenoid operating at such frequencies), the factor $\epsilon^{\prime \prime}(\omega) / \mu^{\prime \prime}(\omega)$ can be very large in diamagnetics, independently of frequency. Let us estimate the latter factor. As is well known, diamagnetism is a relativistic effect of the order of $\beta^{2}(\beta=v / c)$. For the case of forced oscillations of frequency $\omega$, we can estimate $\beta$ as $\beta \sim \omega a / c$ where $a$ is the atomic length scale. Note further that, on the order of magnitude, $\epsilon^{\prime \prime}(\omega) / \mu^{\prime \prime}(\omega) \sim \chi_{e}(\omega) / \chi_{m}(\omega)$, where $\chi_{e}$ and $\chi_{m}$ are the electric and magnetic susceptibilities, respectively. The ratio of the two quantities is estimated in Ref. [8] and the estimate reads

$$
\frac{\chi_{e}(\omega)}{\chi_{m}(\omega)} \sim \frac{6}{\beta^{2}} .
$$

If we use $\beta \sim \omega a / c$ (for forced oscillations), the ratio (6) becomes

$$
\frac{\epsilon^{\prime \prime}(\omega)\left|\mathbf{E}_{\omega}(\mathbf{r})\right|^{2}}{\mu^{\prime \prime}(\omega)\left|\mathbf{H}_{\omega}(\mathbf{r})\right|^{2}} \sim \frac{3}{2}\left(\frac{R}{a}\right)^{2} .
$$

As long as the object under investigation is macroscopic, $R$ $\gg a$ (a factor of 100 is not unreasonable) and the above ratio is always larger than unity. In physical terms, electric losses always dominate over magnetic losses and, even if the latter are negative, the net result is guaranteed to be positive. If this is so, negativity of $\mu^{\prime \prime}$ does not contradict the second law of thermodynamics.

A few comments are necessary at this point. First, the estimate $\beta \sim \omega a / c$ is not accurate at low frequencies. Indeed, this estimate suggests that $\lim _{\omega \rightarrow 0}\left[\chi_{m}(\omega) / \chi_{e}(\omega)\right]=0$. In fact, this ratio approaches some small yet nonzero limit. In natural diamagnetics, the ratio of the real parts of the electric and magnetic susceptibilities (at zero frequency) varies in the range from $10^{-7}$ to $10^{-5}$. The nonzero limit can be understood by considering the fact that electrons in a solid are not at rest but move with the characteristic internal atomic frequencies $\omega_{n m} \neq 0$ even in the strictly static limit. Thus, the zero-frequency diamagnetism is not only a relativistic, but also a quantum effect. The above consideration should not apply to artificial composite materials made of nonmagnetic constituents but designed to posses magnetic properties $[9,10]$. The main reason is that the elementary cells of such materials are macroscopic so that the dynamics of currents and charges inside such cells is fully described by the classical laws of motion. Thus, the formula (8) is expected to be valid for such artificial materials at arbitrarily low frequencies. Second, in the case of artificial materials such as the ones described in Refs. $[9,10]$, the constant $a$ must be understood differently than in the case of natural materials. That is, $a$ in this case is the size of an elementary cell (or the characteristic lattice period) while $R$ is still the overall size of the sample. Obviously, $R$ must always be much larger than $a$ in order for the material to be considered as homogeneous. (Note that, in the above argument, I did not imply that the analysis of Refs. [9,10] is correct and that the artificial materials proposed in these references would indeed have the high-frequency magnetic properties as claimed.)

Finally, the arguments that led me to the claim that $\mu^{\prime \prime}$ can be negative in diamagnetics are based on qualitative estimates. No mathematically rigorous proof of that claim has been given. However, I have demonstrated that there is no mathematically rigorous proof of the opposite claim either. Therefore, the possibility that $\mu^{\prime \prime}(\omega)$ can be negative is, theoretically, open. Of course, it is not possible to make a more definitive statement in the absence of empirical evidence. The arguments presented above suggest that it is not unreasonable to search experimentally for situations in which $\mu^{\prime \prime}$ $<0$, either in naturally occurring diamagnetics or in artificial materials which exhibit a diamagnetic response at low frequencies.

\section{AN ALTERNATIVE EXPRESSION FOR THE HEATING RATE}

So far, all arguments have been based on the expression (1) for the heating rate $q(\mathbf{r})$. This expression is derived by utilizing the conventional form of the Poynting vector in material media. While the conventional formula in nonmagnetic media is not in doubt, an alternative expression for the Poynting vector in magnetically polarizable media has recently been derived $[11,12]$. In particular, as is shown in Ref. [11], this alternative expression results in a modified expression for $q(\mathbf{r})$. The latter predicts exactly the same total absorption $Q$ as is obtained by integrating (1) over the sample volume but a different local distribution of the heating rate. While it may be too early to judge the correctness of this theoretical prediction, it is interesting to consider its implications for the imaginary part of the permeability.

Since this alternative, recently derived expression results in the same integral quantity $Q$, it does not affect any of constraints on $\epsilon(\omega)$ and $\mu(\omega)$ that arise from the condition 
$Q>0$. However, it imposes certain new constraints. It is, therefore, interesting to consider these additional constraints and, in particular, whether the alternative expression for the heating rate requires that $\mu^{\prime \prime}$ be positive.

The derivation of the alternative expression is based on the definition

$$
q=\langle\mathbf{J} \cdot \mathbf{E}\rangle
$$

where

$$
\mathbf{J}=\frac{\partial \mathbf{P}}{\partial t}+c \boldsymbol{\nabla} \times \mathbf{M}
$$

is the total current induced in the medium, including the conductivity current and the currents due to magnetization; I assume that there are no currents or charges which are external to the medium which are often referred to as "free currents."

A straightforward application of the definition (9) with the current given by (10) shows that the heating rate has two contributions which describe (positive or negative) heat generation in the medium volume and at its surface. The terms are denoted by $q^{(V)}$ and $q^{(S)}$, respectively, and are given by

$$
\begin{gathered}
q^{(V)}(\mathbf{r})=\frac{\omega\left|\mathbf{E}_{\omega}(\mathbf{r})\right|^{2}}{8 \pi} \operatorname{Im}[\mu(\omega) \boldsymbol{\epsilon}(\omega)], \\
q^{(S)}(\mathbf{R})=\frac{c}{8 \pi} \operatorname{Re}\left\{[\mu(\omega)-1]\left[\mathbf{E}_{\omega}^{*}(\mathbf{R}) \times \mathbf{H}_{\omega}(\mathbf{R})\right] \cdot \hat{\mathbf{n}}(\mathbf{R})\right\},
\end{gathered}
$$

where $\mathbf{r}$ is a point inside the medium volume, $\mathbf{R}$ is a point on its surface, and $\hat{\mathbf{n}}(\mathbf{R})$ is an outward unit normal to the surface drawn at that point. The total heat generated per unit time by the body is given by

$$
Q=Q^{(V)}+Q^{(S)}=\int_{V} q_{\omega}^{(V)}(\mathbf{r}) d^{3} r+\int_{S} q^{(S)}(\mathbf{R}) d^{2} R,
$$

where the second integral is evaluated over the surface of the body. It is straightforward to show that (13) together with definitions (11) and (12) yields exactly the same total heat per unit time as one would obtain by integrating (1) over the body volume.

If the alternative expression for the heating rate is correct, a new constraint on $\epsilon$ and $\mu$ must be imposed. One might argue that the second law of thermodynamics prohibits cooling of the volume, even if the heat generated at the surface compensates for the cooling and yields a positive net absorption $Q>0$. The constraint that follows from the condition $Q^{(V)}>0$ is

$$
\operatorname{Im}[\epsilon(\omega) \mu(\omega)]>0 .
$$

Detailed thermodynamic considerations are given in Ref. [11] and are not repeated here. Note, however, that one can construct a purely electrodynamic argument in favor of the inequality (14). That is, it is not difficult to see that the expressions for the heating rate (11) and (12) imply that the Poynting vector is given by

$$
\mathbf{S}=\frac{c}{4 \pi} \mathbf{E} \times \mathbf{B}
$$

If this is indeed so, then the direction of the Poynting vector for a plane monochromatic wave propagating in a medium characterized by some arbitrary $\epsilon(\omega)$ and $\mu(\omega)$ coincides with the direction of the phase velocity. On the other hand, the inequality $\operatorname{Im}[\epsilon(\omega) \mu(\omega)]<0$ implies that the wave number $k$ has real and imaginary parts of different signs. Since the real part of $k$ specifies the direction of the phase velocity while the imaginary part of $k$ specifies the direction of exponential decay, the wave exponentially grows in the direction of the Poynting vector if the condition $\operatorname{Im}[\epsilon(\omega) \mu(\omega)]<0$ is satisfied. Such exponential growth appears to be physically impossible.

Thus, sufficient indications exist to accept that (14) is a correct physical constraint on the possible values of $\epsilon(\omega)$ and $\mu(\omega)$, assuming (11) and (12) are correct. This constraint is in addition to all the constraints obtained previously. However, it can be seen that (14) does not require that $\mu^{\prime \prime}>0$. Thus, no contradiction to negativity of the imaginary part of the permeability is obtained by considering the alternative expressions (11) and (12) for the heating rate.

\section{SUMMARY}

The main result of this paper is the finding that, in the case of diamagnetics [1], negativity of the imaginary part of magnetic permeability $\mu(\omega)$ does not contradict the second law of thermodynamics, nor any other conceivable physical constraints. The result applies to naturally occurring diamagnetics as well as to artificial composite structures that exhibit diamagnetic response at low frequencies. Further, if we accept that the imaginary part of $\mu(\omega)$ can be negative, at least in some finite frequency range, the analytical properties of the function $\mu(\omega)$ can be reconciled with the condition $\mu(0)<1$ without undue strain. Specifically, no modification of the Kramers-Kronig relations for $\mu(\omega)$ (such as the modification described in Sec. II) is required in this case.

The conclusion that $\mu^{\prime \prime}(\omega)$ can be negative in diamagnetics is consistent with the conventional expression for the heating rate (1) as well as the alternative expression given by formulas (11)-(13). 
[1] By diamagnetics, I mean here substances with $\mu_{0}=\mu(0)<1$; at larger frequencies, the magnetic susceptibility $\chi_{m}(\omega)$ $=[\mu(\omega)-1] / 4 \pi$ can change sign and become positive as in the case of paramagnetics.

[2] R. A. Depine and A. Lakhtakia, Phys. Rev. E 70, 048601 (2004).

[3] A. L. Efros, Phys. Rev. E 70, 048602 (2004).

[4] T. Koschny, P. Markos, D. R. Smith, and C. M. Soukoulis, Phys. Rev. E 68, 065602(R) (2003).

[5] L. D. Landau and L. P. Lifshitz, Electrodynamics of Continuous Media (Pergamon Press, Oxford, 1984).
[6] V. G. Veselago, Appl. Phys. B: Lasers Opt. 81, 403 (2005).

[7] N. Liu et al., Nat. Photonics 7, 31 (2008).

[8] J. Schwinger, L. L. DeRaad, K. A. Milton, and W. Tsai, Classical Electrodynamics (Perseus Books, Reading, MA, 1998).

[9] J. B. Pendry, A. J. Holden, D. J. Robbins, and W. J. Stewart, IEEE Trans. Microwave Theory Tech. 47, 2075 (1999).

[10] V. A. Podolskiy, A. K. Sarychev, and V. M. Shalaev, Opt. Express 11, 735 (2003).

[11] V. A. Markel, e-print arXiv:0712.0605.

[12] F. Richter, M. Florian, and K. Henneberger, Europhys. Lett. 81, 67005 (2008). 